\title{
Periodic orbits for dissipative twist maps
}

\author{
MARTIN CASDAGLI \\ Department of Mathematics, University of Arizona, Tucson, AZ 85721, USA and \\ Mathematics Institute, University of Warwick, Coventry CV4 7AL, England
}

(Received 15 November 1985 and revised 7 March 1986)

Abstract. We develop simple topological criteria for the existence of periodic orbits in maps of the annulus. These are applied to one-parameter families of dissipative twist maps of the annulus and their attractors. It follows that many of the motions found by variational methods in area preserving twist maps also occur in the dissipative case.

\section{Introduction}

The study of twist maps of the annulus was initiated by Poincaré, in connection with the 3-body problem. In this case the map is area preserving, and Birkhoff [4] was able to use a variational formulation to show that it has many periodic orbits. The variational approach was refined by Aubry [3] and Mather [19], who deduced the existence of well-ordered periodic orbits, and of quasiperiodic orbits which are either invariant curves or Cantor sets.

In this paper we are concerned with the existence of such motions in dissipative twist maps, which have been used as models for various physical processes [2], [7], [14], [21]. As a consequence of the work of Katok [17], [18], and Hall [12], it suffices to consider periodic orbits. In the first part of this paper we use a very simple new technique to deduce the existence of periodic orbits in one-parameter families of dissipative twist maps. The technique is topological in character, and reduces the two dimensional fixed point problem of finding periodic orbits to two essentially one dimensional problems. Our results are reminiscent of those of Chenciner [10], [11] on the degenerate Hopf bifurcation, though our methods are different.

In the second part of this paper we focus attention on the orbit structure of the attractors of dissipative twist maps. It is known that if the map has transverse homoclinic points, then its attracting set contains orbits whose rotation numbers form a non-trivial interval [2], [14]. In [5] Birkhoff showed how to define internal and external rotation numbers for attracting sets of a dissipative twist map, and gave an example where these two numbers enclose a non-trivial interval. By analogy with the area preserving case, it is natural to ask if there are orbits on the invariant set of all rotation numbers lying in this interval. We show that this is indeed the case if the invariant set satisfies an intersection property which we define. Again, topological techniques are used to prove this theorem. Using the topology of the 
annulus, and the geometry of the twist we develop a criterion for the existence of periodic orbits. This criterion represents an improvement on the 'radially translated curve theorem' of Poincaré \& Birkhoff [2].

In contrast to proving the existence of strange attractors (which necessarily satisfy the intersection property), it is easy to prove the existence of invariant sets with the intersection property. We show that every dissipative twist map contains such a set which is weakly attracting.

In $\S 1$ we collect definitions and notation used throughout the paper, and study periodic orbits of parameterized families. In $\S 2$ we prove our results on rotation intervals for invariant sets with an intersection property. In $\S 3$ we prove that every dissipative map of the annulus has an invariant set with the intersection property, which is also weakly attracting.

Acknowledgements. I would like to thank my supervisor, David Rand, for suggesting this problem and bringing Birkhoff's paper [5] to my attention in February 1984. I would also like to thank R. S. MacKay and E. C. Zeeman for useful discussions. Theorem 2.1 provides a different proof of a theorem of P. Le Calvez which, apparently, was achieved nine months earlier than my proof. Whilst Le Calvez attains a stronger result than ours, we believe the simpler route chosen in this paper has independent interest. P. Le Calvez's article will shortly appear in Communications in Mathematical Physics. I would like to thank Le Calvez for pointing out some errors in my original draft. This work was supported by a U.K. Science and Engineering Research Council award, and by the La Jolla Institute Contract, U.S. Department of Energy, DE-AC03-84ER40182.

\section{Families of dissipative twist maps}

Notation. We will use the following notation throughout this paper. $S^{1}$ denotes the unit circle, and $\tilde{A}$ denotes the unit cylinder $S^{1} \times \mathbb{R}$. We define $\tilde{H}$ to be the set of compact connected sets $\tilde{\Lambda}$ separating $S^{1} \times \mathbb{R}$, i.e. $S^{1} \times \mathbb{R}-\tilde{\Lambda}$ consists of two unbounded components $\tilde{\Lambda}_{\text {int }}$ and $\tilde{\Lambda}_{\text {ext }}$, where $\tilde{\Lambda}_{\text {int }}$ (resp. $\tilde{\Lambda}_{\text {ext }}$ ) contains the lower (resp. upper) end of the cylinder. There is a partial order on $\tilde{H}$ defined by $\tilde{\Lambda}<\tilde{\Gamma}$ if $\tilde{\Lambda} \subset \tilde{\Gamma}_{\text {int }}$. An annular region is a set $\tilde{B} \subset \tilde{A}$ with frontier consisting of two disjoint sets $\partial^{+} \tilde{B}, \partial^{-} \tilde{B} \in \tilde{H}$ such that $\partial^{+} \tilde{B}>\partial^{-} \tilde{B}$. A trapping region for a map $\tilde{f}$ of $\tilde{A}$ is a closed annular region $\tilde{B}$ such that $\tilde{f}(\tilde{B}) \subset$ interior $(\tilde{B})$. The lift to $\mathbb{R}^{2}$ of a set $\tilde{S} \subset \tilde{A}$ is denoted by $S$, and all of the above notation lifts in the obvious way.

Definition. A diffeomorphism $f: \mathbb{R}^{2} \rightarrow \mathbb{R}^{2}$ is called a twist map if:

(1) $f$ commutes with $T$ where $T: \mathbb{R}^{2} \rightarrow \mathbb{R}^{2}$ is the unit translation $T(\theta, r)=(\theta+1, r)$. It follows that $f$ is the lift of a unique map $\tilde{f}: \tilde{A} \rightarrow \tilde{A}$ of the cylinder.

(2) $f$ is orientation preserving, and $\tilde{f}$ is end preserving.

(3) There exists $\delta>0$ such that for all $(\theta, r) \in \mathbb{R}^{2}, \partial\left(\pi_{1} f(\theta, r)\right) / \partial r>\delta$, where $\pi_{1}: \mathbb{R}^{2} \rightarrow \mathbb{R}$ is the projection $\pi_{1}(\theta, r)=\theta$.

We call $f$ a dissipative twist map if in addition:

(4) There exists $\lambda \in(0,1)$ such that for all $x \in \mathbb{R}^{2}, 0<$ Det $D f(x) \leq \lambda$.

(5) There exists $M \in \mathbb{R}^{+}$such that for all $N \geq M, S^{1} \times[-N, N]$ is a trapping region for $\tilde{f}$. Note that conditions (1) to (4) do not necessarily imply condition (5). 
In this paper we will be working with a fixed lift $f$ of $\tilde{f}$, so that rotation numbers are uniquely defined.

As a motivating example, consider the two parameter family $f_{\omega, k}$ of dissipative twist maps given by $(1.1)$, where $\lambda \in(0,1)$ is fixed.

$$
f_{\omega, k}(\theta, r)=\left(\theta+\omega+\lambda r-\frac{k}{2 \pi} \sin (2 \pi \theta), \lambda r-\frac{k}{2 \pi} \sin (2 \pi \theta)\right)
$$

If there is a $(\theta, r) \in \mathbb{R}^{2}$ such that $f^{q}(\theta, r)=(\theta+p, r)$ where $p \in \mathbb{Z}, q \in \mathbb{Z}^{+}$, we call $(\theta, r)$ a $p / q$ periodic point. Then define the 'Arnold Tongues' $I_{p / q}$ by (1.2)

$$
I_{p / q}=\left\{(\omega, k) \in \mathbb{R}^{2} \mid f_{\omega, k} \text { has a } p / q \text { periodic orbit }\right\}
$$

We will also be interested in the sets $I_{\sigma}, \sigma \in \mathbb{R}-\mathbb{Q}$ defined by (1.3):

$$
I_{\sigma}=\left\{(\omega, k) \in \mathbb{R}^{2} \mid f_{\omega, k} \text { has an Aubry-Mather set of rotation number } \sigma\right\}
$$

(An Aubry-Mather set is a closed $f, T$ invariant set $M$ which is minimal, and on which $\pi_{1}$ is injective, and $f$ is order preserving: for all $x, x^{\prime} \in M, \pi_{1}(x)<\pi_{1}\left(x^{\prime}\right)$ implies $\pi_{1}(f(x))<\pi_{1}\left(f\left(x^{\prime}\right)\right)$. It follows that $M$ has a well defined rotation number, and is either an invariant curve or a Cantor set [17], [18]).

When $k=0$, the circle $r=0$ is an attracting invariant set for $f_{\omega, k}$. It is also normally hyperbolic, and therefore persists for $|k|$ sufficiently small. It may then be deduced that in this range of $k, I_{p / q} \cap \mathbb{R} \times\{k\} \neq \varnothing$ for all $p / q \in \mathbb{Q}$, and that the $I_{\sigma}$ are curves of the form $\omega=u(k)$, where $u$ is a continuous function. When $|k|$ is large, it is known that smooth invariant curves do not exist [6]. However it is a consequence of the next theorem that all the sets $I_{\omega}, \omega \in \mathbb{R}$ persist for arbitrarily large $k$, though they may overlap. This is illustrated in figure 1 .

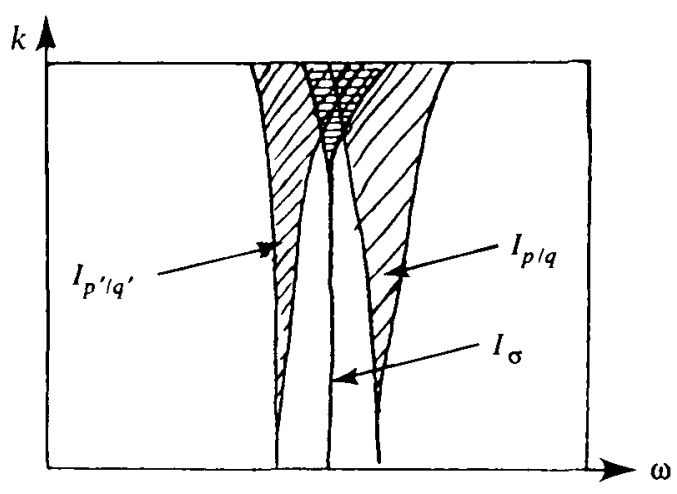

FIGURE 1

THEOREM 1.1. Let $R_{\omega}: \mathbb{R}^{2} \rightarrow \mathbb{R}^{2}$ be the rigid rotation $R_{\omega}(\theta, r)=(\theta+\omega, r)$, and let $f$ be a dissipative twist map. Then for all $p / q \in \mathbb{Q}$ and $\theta_{0} \in \mathbb{R}$, there exists $\omega \in \mathbb{R}$ such that $R_{\omega} \circ f$ has a $p / q$ periodic point on the line $\left\{\theta_{0}\right\} \times \mathbb{R}$. Moreover, for all $\sigma \in \mathbb{R}-\mathbb{Q}$, there exists $\omega \in \mathbb{R}$ such that $R_{\omega} \circ$ f has an Aubry-Mather set of rotation number $\sigma$.

Proof. Fix $\theta_{0} \in \mathbb{R}$, and let $f_{\omega}=R_{\omega} \circ f$. For $\omega_{1}<\omega_{2}$, define the closed set $D_{p q}$ by

$$
D_{p q}=\left\{(\omega, r) \in\left[\omega_{1}, \omega_{2}\right] \times \mathbb{R} \mid \pi_{1} f_{\omega}^{q}\left(\theta_{0}, r\right)=\theta_{0}+p\right\}
$$


and define the continuous function $\Delta: \mathbb{R}^{2} \rightarrow \mathbb{R}^{2}$ by

$$
\Delta(\omega, r)=\left(\omega, \pi_{2} f_{\omega}^{q}\left(\theta_{0}, r\right)-r\right)
$$

We claim that (see figure 2):

(i) $D_{p q}$ contains a connected subset $D_{p q}^{*}$ intersecting both $\left\{\omega_{1}\right\} \times \mathbb{R}$ and $\left\{\omega_{2}\right\} \times \mathbb{R}$.

(ii) There exists $K>0$ such that if $\omega_{1}<-K$ and $\omega_{2}>K$, then $\Delta\left(D_{p q}^{*}\right)$ intersects the set $\mathbb{R} \times\{0\}$ in at least one point $\left(\omega^{*}, 0\right)$.

Then from the definition of $D_{p q}$ and $\Delta$, it follows that $f_{\omega^{*}}$ has a $p / q$ periodic point on the line $\left\{\theta_{0}\right\} \times \mathbb{R}$.

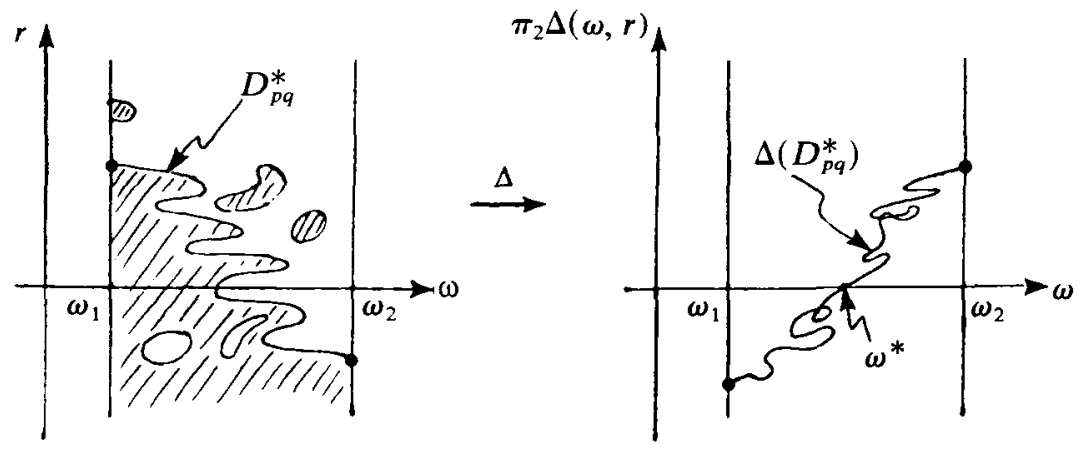

FIGURE 2

To verify (i), consider the open set $O_{1}$ defined by (1.6)

$$
O_{1}=\left\{(\omega, r) \in\left[\omega_{1}, \omega_{2}\right] \times \mathbb{R} \mid \pi_{1} f_{\omega}^{q}\left(\theta_{0}, r\right)<\theta_{0}+p\right\} .
$$

The set $O_{1}$ is shown shaded in figure 2 . From the twist condition, $\tilde{f}_{\omega}$ rotates points arbitrarily much in the positive direction near the upper end of $\tilde{A}$, and arbitrarily much in the negative direction near the lower end of $\tilde{A}$. Thus there exists $N \in \mathbb{R}$ such that $\left[\omega_{1}, \omega_{2}\right] \times[N, \infty) \subset O_{1}^{c}$ and $\left[\omega_{1}, \omega_{2}\right] \times(-\infty,-N] \subset O_{1}$. Let $O_{2}$ be the component of $O_{1}$, containing $\left[\omega_{1}, \omega_{2}\right] \times(-\infty,-N]$ and $O_{3}$ be the component of $\left(\left[\omega_{1}, \omega_{2}\right] \times \mathbb{R}\right)-\mathrm{Cl}\left(O_{2}\right)$ containing $\left[\omega_{1}, \omega_{2}\right] \times[N, \infty)$. Then $O_{3}$ is simply connected, and therefore has a connected frontier $F$, so that $D_{p q}^{*}=D_{p q} \cap F$ has the required properties.

Let $M \in \mathbb{R}$ satisfy condition (5) for the map $f$. It follows that $M$ satisfies condition (5) for all of the maps $f_{\omega}, \omega \in \mathbb{R}$. To verify (ii), we first show that if $\omega$ is sufficiently negative, and $(\omega, r) \in D_{p q}$, then $r \geq M$. Suppose by contradiction that $r<M$. Let $\pi_{1} f_{0}\left(\theta_{0}, M\right)=L$. Then $\pi_{1} f_{0}\left(\theta_{0}, r\right)<L$, and $\pi_{1} f_{\omega}\left(\theta_{0}, r\right)<L+\omega$. Since $f_{\omega}\left(S^{1} \times\{M\}\right)<$ $S^{1} \times\{M\}$, we have by induction that $\pi_{1} f_{\omega}^{q}\left(\theta_{0}, r\right)<q(L+\omega)$. Now choose $\omega$ so that $q(L+\omega)<p$, to contradict the hypothesis that $(\omega, r) \in D_{p q}$. Thus if $\omega_{1}$ is sufficiently negative, it follows from the definition of $M$ that $\pi_{2} f_{\omega_{1}}^{q}\left(\theta_{0}, r\right)<r$, so that $\Delta\left(D_{p q}^{*}\right)$ intersects the lower half-plane. Similarly, if $\omega_{2}$ is sufficiently positive, $\Delta\left(D_{p q}^{*}\right)$ intersects the upper half-plane. Then since $\Delta$ is continuous, and $D_{p q}^{*}$ is connected, it follows that $\Delta\left(D_{p q}^{*}\right)$ is connected, and must intersect the line $\mathbb{R} \times\{0\}$.

It remains to deduce the existence of the Aubry-Mather sets. Following [18], define an orbit

$$
\tilde{\Gamma}=\left\{\left(\theta_{n}, r_{n}\right)=\tilde{f}^{n}\left(\theta_{0}, r_{0}\right) \mid n \in \mathbb{Z}\right\} \subset \tilde{A}
$$


to be a special orbit if there exists a homeomorphism $g: S^{1} \rightarrow S^{1}$ such that $g^{n}\left(\theta_{0}\right)=\theta_{n}$ for all $n \in \mathbb{Z}$. $\tilde{\Gamma}$ has a well defined rotation number $\rho(\tilde{\Gamma})=\rho(g)$. A result of Hall [12] asserts that if $\tilde{f}$ is a twist map, and has a $p / q$ periodic orbit, then $\tilde{f}$ has a special orbit of rotation number $p / q$. Hence from the first part of the theorem, given any $\sigma \in \mathbb{R}-\mathbb{Q}$, and a sequence of rationals $\left\{p_{m} / q_{m} \mid m \in \mathbb{Z}^{+}\right\}$converging to $\sigma$, there exists a sequence of reals $\left\{\omega_{m} \mid m \in \mathbb{Z}^{+}\right\}$such that $\tilde{f}_{\omega_{m}}$ has a special orbit $\tilde{\Gamma}_{m}=$ $\left\{\tilde{f}_{\omega_{m}}^{n}\left(\theta_{m}, r_{m}\right) \mid n \in \mathbb{Z}\right\}$ of rotation number $p_{m} / q_{m}$. By condition (5) the sets $\tilde{\Gamma}_{m}, m \in \mathbb{Z}^{+}$ all lie in the compact subset $S^{1} \times[-M, M]$ of $S^{1} \times \mathbb{R}$, and the $\omega_{m}, m \in \mathbb{Z}^{+}$are bounded. Thus, if necessary by going to a subsequence, the sequence $\left\{\left(\theta_{m}, r_{m}, \omega_{m}\right) \mid m \in \mathbb{Z}^{+}\right\}$ can be chosen to converge to a point $(\theta, r, \omega)$ as $m \rightarrow \infty$. If we can show that $\tilde{\Gamma}=\left\{\tilde{f}_{\omega}^{n}(\theta, r) \mid n \in \mathbb{Z}\right\}$ is a special orbit of rotation number $\sigma$, the proof of theorem 1.1 will be complete, since the $\omega$-limit set of the orbit $\tilde{\Gamma}$ is an Aubry-Mather set of rotation number $\sigma$.

The proof is now almost identical to that in [18]. The function $\tilde{\psi}_{m}: \pi_{1} \tilde{\Gamma}_{m} \rightarrow[a, b]$, which assigns $\pi_{2} \tilde{f}_{\omega_{m}}^{n}\left(\theta_{m}, r_{m}\right)$ to $\pi_{1} \tilde{f}_{\omega_{m}}^{n}\left(\theta_{m}, r_{m}\right)$ is Lipshitz, with Lipshitz constant $L$ independent of $m$. Extend $\tilde{\psi}_{m}$ by linear interpolation to a map $\tilde{\psi}_{m}^{\prime}: S^{1} \rightarrow[a, b]$, also with Lipshitz constant $L$. The space $\tilde{\mathbb{L}}$ of Lipshitz maps from $S^{1}$ to $[a, b]$ with Lipshitz constant $L$ is compact in the topology of uniform convergence. Thus, if necessary by going to a subsquence, we can find $\tilde{\psi}^{\prime} \in \tilde{\mathbb{L}}$ such that $\lim _{m \rightarrow \infty} \tilde{\psi}_{m}^{\prime}=\tilde{\psi}^{\prime}$. By the definition of convergence in $\tilde{\mathbb{L}}$, we have $\tilde{\Gamma} \subset \operatorname{graph}\left(\tilde{\psi}^{\prime}\right)$. The circle map $g=\pi_{1} \circ \circ \circ\left(\mathrm{id} \times \tilde{\psi}^{\prime}\right)$ is order preserving, so that $\tilde{\Gamma}$ is special. By construction, $\lim _{m \rightarrow \infty} \rho\left(\tilde{\Gamma}_{m}\right)=\sigma$, so that for every $k$, the order of the first $k$ points for the rigid rotation by $R_{\sigma}$ is the same as the rotation by $R_{\rho\left(\tilde{\Gamma}_{m}\right)}$, for sufficiently large $m$. Thus since $\rho(\tilde{\Gamma})$ is well defined, it follows that $\rho(\tilde{\Gamma})=\sigma$.

\section{Rotation Intervals}

In this section we explore the possibility of periodic orbits coexisting on invariant sets of a dissipative twist map $f$. Let $\Gamma \in \mathbb{H}$ be an invariant set for $f$. Then as in [5], we associate with $\Gamma$ two real numbers, its internal and external rotation numbers $\rho_{\text {int }}(\Gamma)$ and $\rho_{\text {ext }}(\Gamma)$, defined as follows. Let $L=\left\{(\theta, r) \in \mathbb{R}^{2} \mid \theta \in \mathbb{R}, r=r_{0}\right\}$ be a horizontal line lying above $\Gamma$. Denote by $\Gamma^{\prime}$ those points $x$ in $\Gamma$ for which the vertical line joining $x$ to $L$ contains no other points of $\Gamma$, and let $\pi$ be the (bijective) vertical projection from $L$ to $\Gamma^{\prime}$. Then $\rho_{\text {ext }}(\Gamma)$ is defined to be $-\rho(g)$ where $\rho(g)$ is the rotation number of the map $g: L \rightarrow L$ defined by $g(\theta)=\pi^{-1} \circ f^{-1} \circ \pi(\theta)$. It can be shown that $f^{-1}\left(\Gamma^{\prime}\right) \subset \Gamma^{\prime}$, so that $g$ is well defined [5]. Moreover it can be seen that $g$ is the lift of a circle map, and that $g$ is monotonic, so that $\rho(g)$ is well defined [20]. Similarly, $\rho_{\text {int }}(\Gamma)$ is defined by considering pre-images of points on $\Gamma$ vertically accessible from a horizontal line lying below $\Gamma$.

In [5] Birkhoff gave an example of a dissipative twist map with an invariant set $\Gamma \in \mathbb{H}$ for which $\rho_{\text {int }}(\Gamma) \neq \rho_{\text {ext }}(\Gamma)$. By analogy with area preserving twist maps and non-invertible circle maps [16], it is natural to ask whether $f$ has $p / q$ periodic orbits for all $p / q \in\left[\rho_{\text {int }}(\Gamma), \rho_{\text {ext }}(\Gamma)\right]$. That this need not be the case is shown in [14]: there are examples of dissipative twist maps constructed as Poincaré return maps of differential equations which have the following orbit structure. Referring to figure 3 , 
in which $a b$ is to be identified with $c d$, the map $f$ has an invariant curve $C$ with rotation number $\omega \neq 0$, and an unstable hyperbolic fixed point $x$, with stable and unstable manifolds $W^{s}(x), W^{u}(x)$. All other points are either attracted to another fixed point $y$ (shaded region), or are attracted to the invariant curve $C$. Then the invariant set $\Gamma=C \cup W^{u}(x)$ has $\left[\rho_{\text {int }}(\Gamma), \rho_{\text {ext }}(\Gamma)\right]=[0, \omega]$, but there are no $p / q$ periodic orbits for $p / q \in(0, \omega)$.

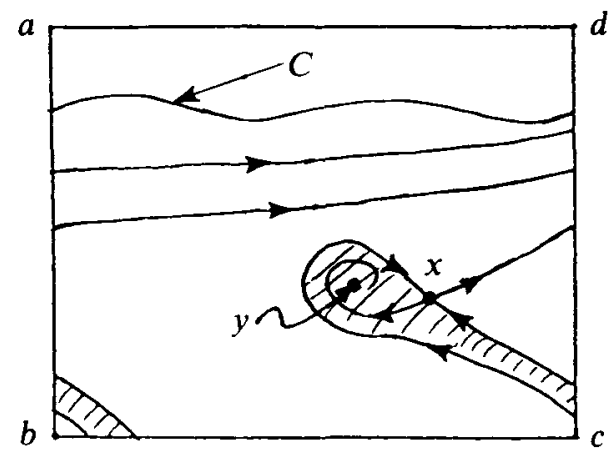

FIGURE 3

The above example motivates the following definition.

Definition. We say that an $f$-invariant set $\Gamma \in \mathbb{M}$ has the intersection property if given any set $C \in \mathbb{H}$ with $C \cap \Gamma \neq \varnothing$, then $C \cap f(C) \neq \varnothing$.

Remark. If $\Gamma \in \mathbb{H}$ has a dense orbit, then it will necessarily satisfy the intersection property.

The next theorem states that if an invariant set has the intersection property then it possesses an 'interval of rotation numbers'.

THEOREM 2.1 Let $f$ be a twist map, and $\Gamma$ be an invariant set with the intersection property. Then $f$ has a $p / q$ orbit for all $p / q \in\left[\rho_{\text {int }}(\Gamma), \rho_{\text {ext }}(\Gamma)\right]$, and an Aubry-Mather set of rotation number $\omega$ for all $\omega \in\left[\rho_{\mathrm{int}}(\Gamma), \rho_{\mathrm{ext}}(\Gamma)\right]$.

The essential idea in the proof of theorem 2.1 is to consider the sets $C_{p q}, p \in \mathbb{Z}$, $q \in \mathbb{Z}^{+}$, defined by $(2.1)$

$$
C_{p q}=\left\{(\theta, r) \mid \pi_{1} f^{q}(\theta, r)=\theta+p\right\}
$$

We first prove a lemma which gives a criterion for the existence of periodic points in terms of the sets $C_{p q}$. Define $P_{p q}$ to be the set of $p / q$ periodic points of $f$.

LEMMA 2.1 Let $f$ be a twist map, then for all $p \in \mathbb{Z}, q \in \mathbb{Z}^{+}$we have $P_{p q}=C_{p q} \cap f\left(C_{p q}\right)$. Proof. Evidently $P_{p q} \subset C_{p q} \cap f\left(C_{p q}\right)$. To show the converse, take a point $\left(\theta_{0}, r_{0}\right)$ in $C_{p q} \cap f\left(C_{p q}\right)$ and consider its orbit $\left\{\left(\theta_{n}, r_{n}\right) \mid n \in \mathbb{Z}\right\}$. By hypothesis $\theta_{q}=\theta_{0}+p$ and 
$\theta_{q-1}=\theta_{-1}+p$, and it remains to show that $r_{q}=r_{0}$. Since $f$ is a twist map, $\left(\theta_{0}, r_{0}\right)$ is given by the unique intersection of the bi-infinite vertical line through $\theta_{0}$ with the image of the bi-infinite vertical line through $\theta_{-1}$. But $f$ commutes with the translation $T^{p}$, hence the same process applied to the bi-infinite vertical lines through $\theta_{q}$ and $\theta_{q-1}$ must yield $r_{q}=r_{0}$. Thus $C_{p q} \cap f\left(C_{p q}\right) \subset P_{p q}$ as required.

Remark. After proving lemma 2.1, it was pointed out to us that similar ideas are used in the 'radially translated curve theorem' of Poincaré \& Birkhoff [1]. In fact they derive $P_{p q}=C_{p q} \cap f^{q}\left(C_{p q}\right)$, but this is only true for sufficiently small non-linearity (dependent on $q$ ). In this sense lemma 2.1 is a more powerful criterion for the existence of periodic orbits.

The next lemma indicates that lemma 2.1 will be useful, by establishing a topological property of the sets $C_{p q}$.

LEMMA 2.2 Let $f$ be a twist map and take $p \in \mathbb{Z}, q \in \mathbb{Z}^{+}$. Then the set $C_{p q}$ is non-empty, and has a component $C_{p q}^{*}$ in $\mathrm{H}$.

Proof. Consider the open set $O_{1} \subset \mathbb{R}^{2}$ defined by (2.2)

$$
O_{1}=\left\{(\theta, r) \mid \pi_{1} f^{q}(\theta, r)<\theta+p\right\}
$$

From the twist condition, $\tilde{f}$ rotates points arbitrarily much in the positive direction near the upper end of $\tilde{A}$, and arbitrarily much in the negative direction near the lower end of $\tilde{A}$. Thus there exists $N \in \mathbb{R}$ such that $\mathbb{R} \times[N, \infty) \subset O_{1}^{c}$ and $\mathbb{R} \times$ $(-\infty,-N] \subset O_{1}$. Let $O_{2}$ be the component of $O_{1}$ containing $\mathbb{R} \times(-\infty,-N]$ and $O_{3}$ be the component of $\mathbb{R}^{2}-\mathrm{Cl}\left(\mathrm{O}_{2}\right)$ containing $\mathbb{R} \times[N, \infty)$. Then $\mathrm{O}_{3}$ is simply connected, and therefore has a connected frontier $C_{p q}^{*} \subset C_{p q}$. Since $O_{1}$ is invariant under the translation $T$, so is $C_{p q}^{*}$, and it follows that $C_{p q}^{*} \in \mathbb{H}$.

Proof of theorem 2.1 Let $p / q \in\left[\rho_{\text {int }}(\Gamma), \rho_{\text {ext }}(\Gamma)\right]$. We first show that $C_{p q}^{*} \not \subset \Gamma_{\text {ext }}$. From the definition of $\rho_{\text {ext }}(\Gamma)$, there exists a point $y=(\theta, r) \in \Gamma^{\prime}$ satisfying (2.3).

$$
\pi_{1} f^{-q}(y) \leq \theta_{0}-q \rho_{\mathrm{ext}}(\Gamma)
$$

Let $L$ be the vertical line through $y$ given by $L=\left\{(\theta, r) \in \mathbb{R}^{2} \mid \theta=\theta_{0}, r \geq r_{0}\right\}$, and consider its image under $f^{-q}$. If $C_{p q}^{*}$ were a subset of $\Gamma_{\text {ext }}$, then $f^{-q}(L)$ would necessarily intersect it at some point $z=(\theta, r)$. Since $f$ is a twist map, one deduces that $\theta<\pi_{1} f^{-q}(y)$ [13]. Then using inequality (2.3), and $p / q \leq \rho_{\text {ext }}(\Gamma)$, we derive $\pi_{1} f^{q}(z)>\pi_{1}(z)+p$, which contradicts $z \in C_{p q}^{*}$. Hence $C_{p q}^{*} \not \subset \Gamma_{\text {ext }}$.

Similarly $C_{p q}^{*} \not \subset \Gamma_{\text {int }}$, and we obtain $C_{p q}^{*} \cap \Gamma \neq \varnothing$. By lemma $2.2, C_{p q}^{*} \in \mathbb{H}$, and since $\Gamma$ is assumed to satisfy the intersection property, we have $C_{p q}^{*} \cap f\left(C_{p q}^{*}\right) \neq \varnothing$. Thus by lemma 2.1, we conclude that $f$ has a $p / q$ periodic point. As in theorem 1.1 , the existence of the Aubry-Mather sets follows directly from the results of Hall [12], and Katok [17].

\section{Sets with the intersection property}

In this section we construct invariant sets with the intersection property for a dissipative twist map. We will also be interested in the attracting properties of these sets; to be precise we make some definitions. 
Definition. We say that a closed $f$-invariant set $\Gamma$ is weakly attracting if for any neighbourhood $N$ of $\Gamma$, there is a neighbourhood $M$ of $\Gamma$ contained in $N$ such that $f(M) \subset M$. If in addition $M$ may be chosen so that $\Gamma=\bigcap_{n=0}^{\infty} f^{n}(M)$, we say that $\Gamma$ is attracting.

Let $B$ be a trapping region for a dissipative twist map $f$. Then the invariant set $\Gamma(B)=\bigcap_{n=0}^{\infty} f^{n}(B)$, is attracting and is a member of $\mathbb{H}$.

Definition. The set $\beta(f)=\mathrm{cl}\left(\Gamma_{\mathrm{int}}(B)\right) \cap \mathrm{cl}\left(\Gamma_{\mathrm{ext}}(B)\right)$ is called the Birkhoff attractor of $f$.

It may be verified that $\beta(f)$ is well defined (i.e. independent of the choice of $B$ ), is a member of $\mathbb{H}$, and satisfies the intersection property [8]. However $\beta(f)$ need not be even weakly attracting [9].

We propose to focus attention instead on another set which turns out to have some nicer properties. Define $\mathbb{H}^{ \pm}$by $\mathbb{H}^{+}=\left\{C \in \mathbb{H} \mid f(C) \subset C_{\text {ext }}\right\}$ and $\mathbb{H}^{-}=$ $\left\{C \in \mathbb{W} \mid f(C) \subset C_{\text {int }}\right\}$. Since $f$ is dissipative, given $C^{ \pm} \in \mathbb{H}^{ \pm}$, we have $C^{+}<C^{-}$, so that $C^{ \pm}$bound an annular region $R\left(C^{-}, C^{+}\right)$. The set $\alpha(f)$ that we are interested in is defined to be the intersection over all annular trapping regions, i.e. $\alpha(f)=$ $\bigcap R\left(C^{-}, C^{+}\right)$where the intersection runs over all $C^{ \pm}$in $B^{ \pm}$.

THEOREM 3.1 The set $\alpha(f)$ is a member of $\mathrm{H}$, satisfies the intersection property, and is weakly attracting.

Proof. To exploit compactness properties, we work in $\tilde{A}$, but for ease of notation, drop the superscript . We verify the required three properties for $\alpha(f)$.

(i) $\alpha(f) \in \mathbb{H}$. By construction $\alpha(f)$ is a closed and bounded set, thus is compact. The complement of $\alpha(f)$ is given by $R_{\text {int }} \cup R_{\text {ext }}$ where $R_{\text {int }}=\bigcup_{C \in \mathrm{H}^{+}} C_{\text {int }}$ and $R_{\text {ext }}=$ $\bigcup_{C \in \mathcal{H}^{-}} C_{\text {ext }}$. Since $f$ is dissipative, we deduce that $R_{\text {int }} \cap R_{\text {ext }}=\varnothing$, and that $\alpha(f)$ disconnects $\tilde{A}$ into these two components only. It remains to show that $\alpha(f)$ is connected. From its definition, $\alpha(f)$ is seen to be equivalent to a nested intersection of compact connected sets, and is therefore connected [15].

(ii) $\alpha(f)$ satisfies the intersection property. Let $C \in \mathbb{H}$ be such that $\alpha(f) \cap C \neq \varnothing$. If we can show that $C$ is not a member of $\mathbb{H}^{+} \cup \mathbb{H}^{-}$, then it follows that $C \cap f(C) \neq \varnothing$ as required. Suppose by contradiction that $C \in \mathbb{H}^{-}$, and take a point $x \in \alpha(f) \cap C$. But then $x$ would lie above $f(C)$ which itself is a member of $\mathbb{H}^{-}$. This contradicts the hypothesis that $x \in \alpha(f)$. Similarly, we cannot have $C \in \mathbb{N}^{+}$.

(iii) $\alpha(f)$ is weakly attracting. Take any neighbourhood $N$ of $\alpha(f)$. We must show that there exists a neighbourhood $M$ of $\alpha(f)$ such that $M \subset N$ and $f(M) \subset$ interior $(M)$. Assume, by going if necessary to a subset of $N$, that $N$ is an annular neighbourhood of $\alpha(f)$. We construct $M$ as follows. Take a point $x$ in $\partial^{+} N$. Then $x$ is not in $\alpha(f)$, so there exists $C(x) \in \mathbb{H}$ such that $x \in C(x)_{\text {ext }}$. Let $\partial^{+} N(x)$ be a non-empty open connected subset of $\partial^{+} N$ containing $x$ such that $C(x) \cap \partial^{+} N(x)=$ $\varnothing$. Since $\partial^{+} N$ is compact, $\partial^{+} N=\bigcup_{i=1}^{n} N\left(x_{i}\right)$ for some $n$ and $x_{1}, \ldots, x_{n}$ in $\partial^{+} N$. Consider the set $S=\bigcap_{i=1}^{n} C\left(x_{i}\right)_{\text {int }}$. By construction $S \cap \partial^{+} N=\varnothing$, and $f(S) \subset S$. Then $\partial^{+} M$ is taken to be the frontier of $S$. Defining $\partial^{-} M$ similarly, the set $M$ is taken to be the open set bounded by $\partial^{ \pm} M$. By construction $M \subset N$, and $f(M) \subset$ interior $(M)$. 


\section{REFERENCES}

[1] V. I. Arnold \& A. Avez. Ergodic Problems of Classical Mechanics. pp. 88-90. Benjamin: New York 1968.

[2] D. G. Aronson, M. A. Chory, G. R. Hall \& R. P. McGehee. Bifucations from an invariant circle for two-parameter families of maps of the plane: A computer assisted study. Commun. Math. Phys. 83 (1982), 303-354.

[3] S. Aubry \& P. L. Le Daeron. The discrete Frenkel-Kontorova model and its extensions. Physica 8D (1983), 381-422.

[4] G. D. Birkhoff. On the periodic motions of dynamical systerns. Acta Mathematica 50, 359-379. Reprinted in Collected Mathematical Papers, Vol. II, American Math. Soc., New York 1950.

[5] G. D. Birkhoff. Sur quelques courbes fermées remarkables. Bull. Soc. Math. de France, 60 (1932), 1-26. Reprinted in Collected Mathematical Papers, Vol. II, American Math. Soc., New York 1950.

[6] T. Bohr. A bound for the existence of invariant circles in a class of two-dimensional dissipative maps. Preprint, Cornell University (1984).

[7] T. Bohr, P. Bak \& M. H. Jensen. Transition to chaos by interaction of resonances in dissipative systems. Preprint, Cornell University (1984).

[8] P. Le Calvez. Existence d'orbites quasi-periodiques dans les attracteurs de Birkhoff. Preprint, Université Paris, Orsay (1985).

[9] P. Le Calvez. Private communication.

[10] A. Chenciner. Sur un énoncé dissipative du théorèm géometrique de Poincaré-Birkhoff. C.R.A.S. t294, Série I (1982), 243-245.

[11] A. Chenciner. Orbites périodiques et ensembles de Cantor invariant d' Aubry-Mather au voisinage d'une bifurcation de Hopf dégénérée de difféomorphismes de $\mathbb{B}^{2}$. C.R.A.S. t297, Série I (1983), 465-467.

[12] G. R. Hall. A topological version of a theorem of Mather on twist maps. Preprint, University of Wisconsin, Madison (1983).

[13] M. Herman. Sur les courbes invariantes par les difféomorphismes de l'anneau. Vol. I, Ch. 1. Astérisque, 103-104 (1983).

[14] K. Hockett \& P. Holmes. Josephson's junction, annulus maps, Birkhoff attractors, horseshoes and rotation sets. Ergod. Th. \& Dynam. Sys. 6 (1986), 205-239.

[15] J. G. Hocking \& G. S. Young. Topology. Addison-Wesley: Reading 1961.

[16] R. Ito. Rotation sets are closed. Math. Proc. Comb. Phil. Soc. 89 (1981), 107-111.

[17] A. Katok. Some remarks on Birkhoff and Mather twist map theorems, Ergod. Th. \& Dynam. Sys. 2 (1982), 185-194.

[18] A. Katok. Periodic and quasiperiodic orbits for twist maps. In Proceedings, Sitges 1982 L. Garrido (ed.), Springer-Verlag, Berlin.

[19] J. N. Mather. Existence of quasiperiodic orbits for twist homomorphisms. Topology 21 (1982), 457-467.

[20] Z. Nitecki. Differentiable Dynamics. M.I.T. Press: 1971.

[21] S. Ostlund, D. Rand, J. Sethna \& E. Siggia. Universal properties of the transition from quasiperiodicity to chaos in dissipative systems. Physica 8D (1983), 303-342. 